\title{
Successful Outcome of Severe Fibrous Dysplasia of the Tibia in a Patient with Mccune-Albright Syndrome, Treated Using a Combined Ortho-Plastic Multidisciplinary Approach
}

\author{
Samena Chaudhry ${ }^{1 *}$, Saqib Noor ${ }^{2}$, Rob Warner ${ }^{3}$ and Paul Fenton ${ }^{4}$ \\ ${ }^{1}$ Senior Clinical Fellow in Paediatric Orthopaedics Derby Royal Hospital, UK \\ ${ }^{2}$ Orthopaedic Registrar West Midlands, UK \\ ${ }^{3}$ Consultant - Plastic Surgeon, Queen Elizabeth Hospital Birmingham, UK \\ ${ }^{4}$ Consultant Trauma and Orthopaedic Surgeon, Queen Elizabeth Hospital Birmingham, UK
}

*Corresponding author: Samena Chaudhry, Senior Clinical Fellow in Paediatric Orthopaedics, Royal Derby Hospital, UK

\begin{abstract}
McCune-Albright syndrome is a genetic disease, defined by a clinical triad of polyostotic fibrous dysplasia of bone, café-au-lait skin spots, and precocious puberty. The case of a young man with McCune-Albright syndrome and fibrous dysplasia resulting in a severe tibial deformity is presented.

Due to previous surgeries as a child, and subsequent complications with ongoing infection and soft tissue breakdown, the patient was keen to seek any reconstructive option with a chance of limb salvage.

Multilevel, corrective tibial osteotomies were carried out by the orthopaedic team, followed by an acute deformity correction of the tibia, stabilised with an intramedullary tibial nail. This was followed by a soft tissue debridement and anterolateral thigh free flap by the plastic surgical team to cover the previously infected and broken down soft tissue over the anterior aspect of the tibia. The 10 hour surgery was completed without complication.
\end{abstract}

A combined multidisciplinary approach to this rare disease and associated severe deformity has demonstrated an excellent outcome at one year with complete healing of the tibia. The patient is fully mobile and is pain free.

\section{Introduction}

McCune-Albright syndrome is a genetic disease, defined by a clinical triad of polyostotic fibrous dysplasia of bone, café-au-lait skin spots, and precocious puberty. In addition, multiple endocrinopathies including hyperthyroidism, growth hormone excess and Cushing syndrome have been associated with the condition. It is a rare disease with estimated prevalence between $1 / 100,000$ and $1 / 1,000,000[1,2]$.

Fibrous dysplasia can affect multiple sites in the axial and appendicular skeleton, with abnormalities in the craniofacial region causing facial asymmetry and in long bones, causing pain, deformity and occasionally pathological fracture. Radiographs typically demonstrate expansile lesions with endosteal scalloping and thinning of the cortex, demonstrating a classic "ground glass" appearance. The areas most commonly involved are the proximal femora and skull base. The sites of fibrous dysplasia involvement are established early in childhood, with $90 \%$ of the total body skeletal disease burden being established by age $15[2,3]$. The use of intramedullary devices is advised for all lower limb reconstructions for fracture or deformity although the femur is more commonly affected than the tibia $[4,5]$.

\section{Case}

A 29-year-old male with McCune-Albright syndrome, presented to the Queen Elizabeth Hospital in 2015, with severe disability and deformity of the right tibia as a result of fibrous dysplasia (Figure 1). He was under the endocrinologist and had café au lait spots with a history of precocious puberty.

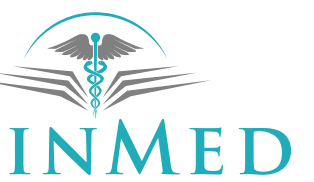

INTERNATIONAL LIBRARY

Citation: Chaudhry S, Noor S, Warner R, Fenton P (2019) Successful Outcome of Severe Fibrous Dysplasia of the Tibia in a Patient with Mccune-Albright Syndrome, Treated Using a Combined Ortho-Plastic Multidisciplinary Approach. Trauma Cases Rev 5:070. doi.org/10.23937/2469-5777/1510070 Accepted: March 23, 2019: Published: March 25, 2019

Copyright: (C) 2019 Chaudhry S, et al. This is an open-access article distributed under the terms of the Creative Commons Attribution License, which permits unrestricted use, distribution, and reproduction in any medium, provided the original author and source are credited. 

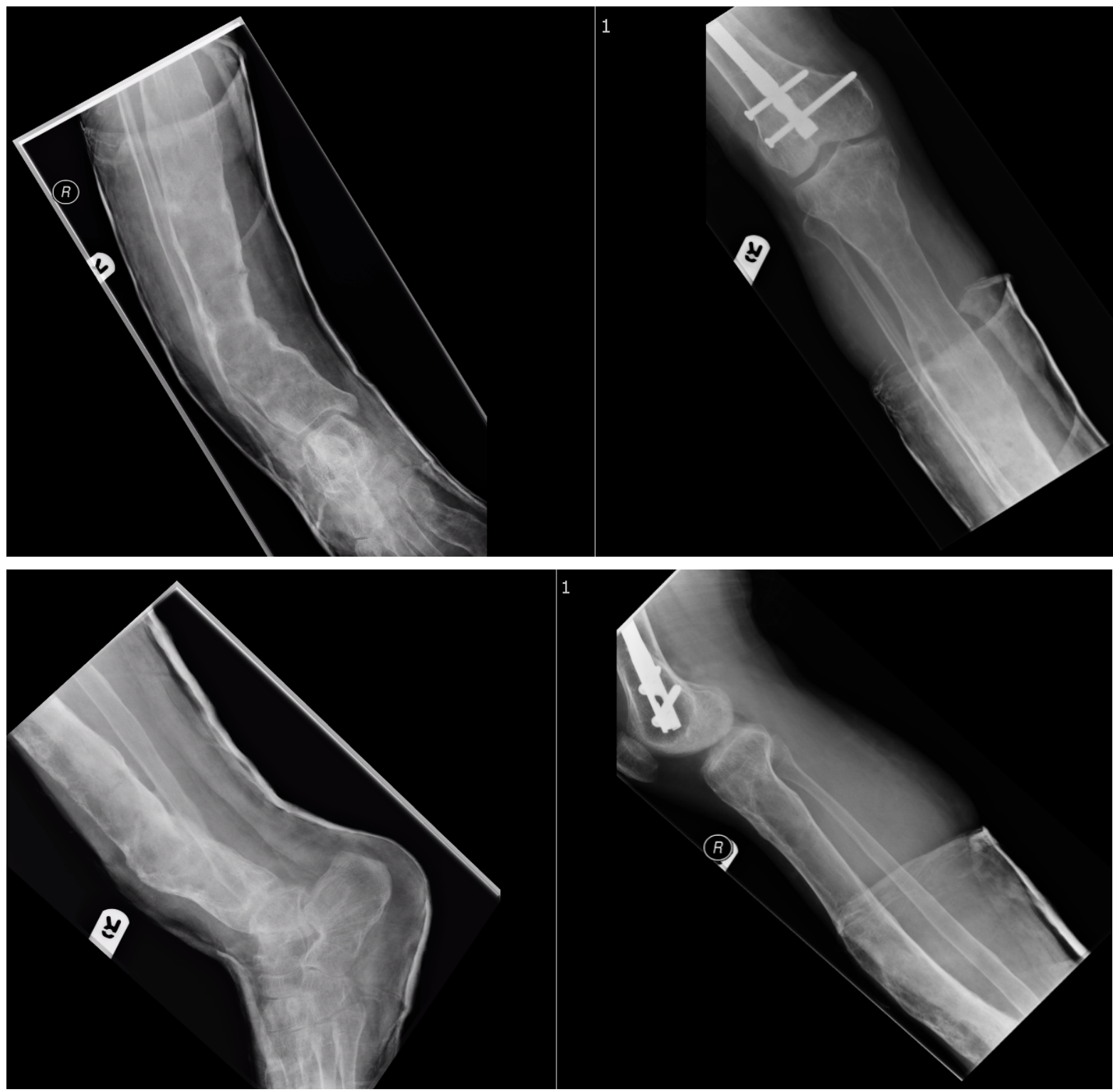

Figure 1: X-rays showing the tibial deformity.

Following previous surgeries as a child, and subsequent complications including ongoing infection and soft tissue breakdown, the patient now complained of worsening deformity, wound discharge and inability to mobilise without crutches, when previously independently mobile.

All reconstructive options were fraught with difficulty and posed significant complications, including nerve injury, severe haemorrhage, deep infection or failure of the correction. With this in mind, the possibility of a primary amputation was also offered to the patient. After a multidisciplinary collaboration, including plastic, orthopaedic, radiological and medical specialities, and counselling of the patient, reconstructive surgery was offered.

In January 2016, combined ortho-plastic surgery was performed. Multilevel, corrective tibial osteotomies were carried out, followed by an acute deformity correction of the tibia, stabilised with an intramedullary tibial nail (orthopaedics). This was followed by a soft tissue debridement and anterolateral thigh free flap (plastics) to cover the previously infected and broken down soft tissue over the anterior aspect of the tibia. The 10 hour surgery was completed without complication.

\section{Methods}

The debridement was done with plastic and orthopaedic surgeons combined. A supra-patellar nail was inserted (synthes) under image intensifier. Due to the proximal tibial apex posterior deformity the entry point was noted to be somewhat anterior. However an osteotomy close to the knee joint was avoided in order to prevent compromise to a future below knee amputation. A guidewire was inserted with multiple blocking screws to centralise wire.

An osteotomy at mid shaft and distal tibia was then performed with a $2.5 \mathrm{~mm}$ drill followed by osteotomes. The cans, was reamed to $11.5 \mathrm{~mm}$ in order to insert the $10 \times 375 \mathrm{~mm}$ expert tibial nail The proximal locking via jig but freehand distal locking. 


\section{Reconstruction with a free antero-lateral thigh flap}

Debridement: The Wound over the anterior aspect of right distal leg over the area of tibial deformity was excised and surrounding unhealthy skin. The SPY?? tissue perfusion system was used to aid debridement and further skin excised medially. The surgical wound measured $15 \times 9 \mathrm{~cm}$.

Vessel preparation: The orientation of wound, a previous medial local flap and to avoid proximal dissection given extensive vasculature proximally which had been identified on pre-op angiogram, helped decide to use the posterior tibial vessels more distally. The posterior tibial vessels were identified and prepared at medial malleolus level. The tibial nerve was released in the tarsal tunnel.

ALT flap raising: We used Standard ALT marking on left thigh with the SPY system used to aid perforator identification. An ALT free flap was raised from left thigh based on single perforator. The course of the perforator was intramuscular through Vastus Lateralis. Part of the muscle was divided to aid dissection. The pedicle was dissected proximally and the motor nerves preserved.
Anastomosis: We performed an end-to-end anastomosis to the posterior tibial vessels. The anastomosis was done distally with good antegrade flow. Vein (RMW, KK) $2.5 \mathrm{~mm}$ venous coupler Artery (KK) - 9/0 Ethilon Was used for the repair and The ischaemia time was $60 \mathrm{~min}$.

Donor site closure: A partial wound closure was performed with 2/0, 3/0 Vicryl and 3/0 Monocryl. A split skin graft was then SSG harvested from antero-medial left thigh. This was meshed 1.5:1. This SSG was applied to the centre of ALT donor site wound and secured with tissue glue.

Flap inset and wound closure: The Flap was inset into defect and tip of flap used to cover anastomosed vessels. We closed in layers using 3/0, 4/0 Vicryl and 4/0 Vicryl Rapide, Blue gauze, Wool and Crepe bandage was applied to the wound and 6/0 Prolene to mark perforator doppler site.

\section{Results}

At 3 and 9 months post surgery, the patient reported no significant problems and at latest follow up, the soft tissue and tibia have gone on to heal clinically (Figure 2) and radiologically (Figure 3). The patient is delighted with the results and is now mobilising unaided once
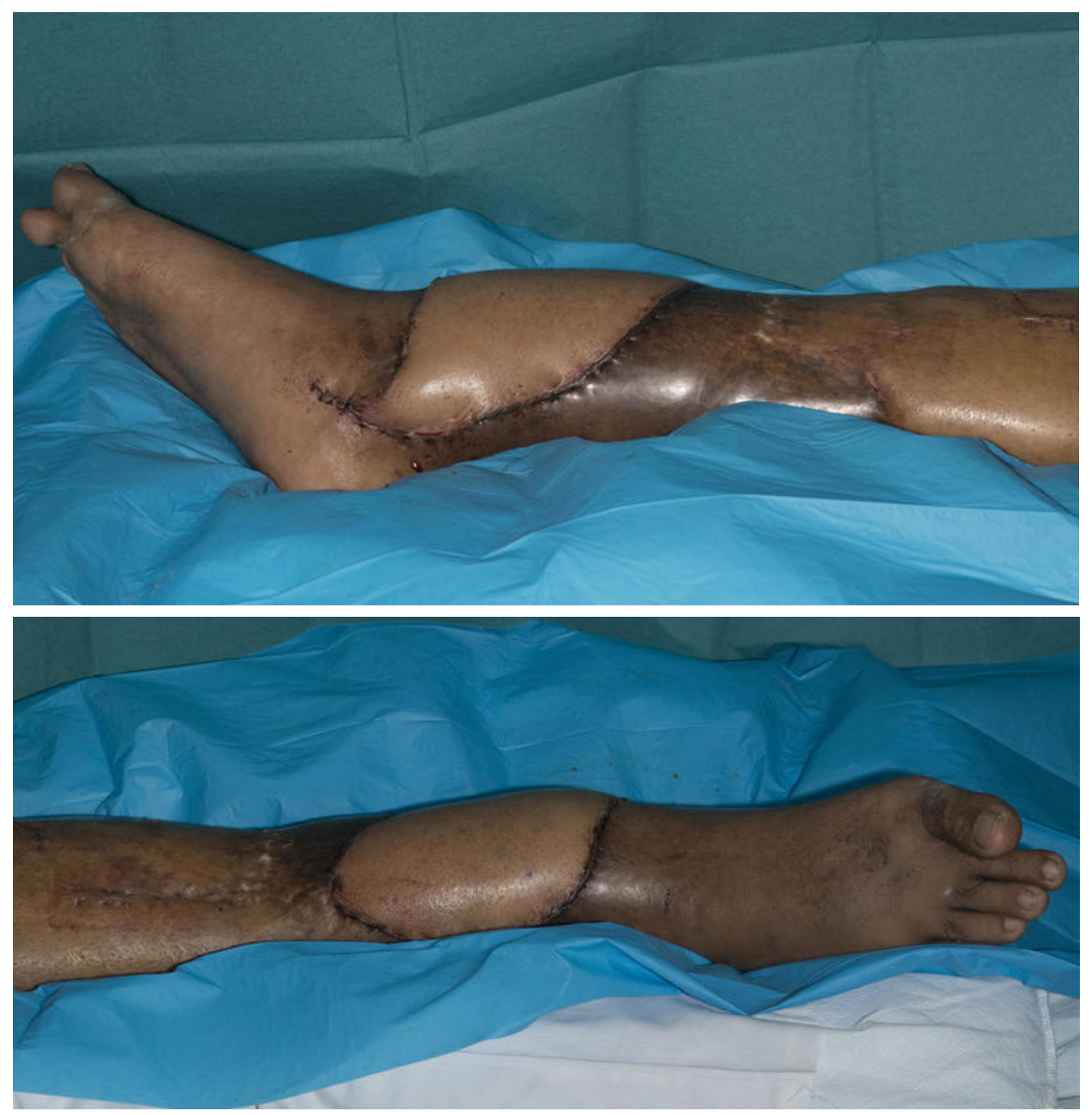

Figure 2: Post corrective surgery and free flap reconstruction. 


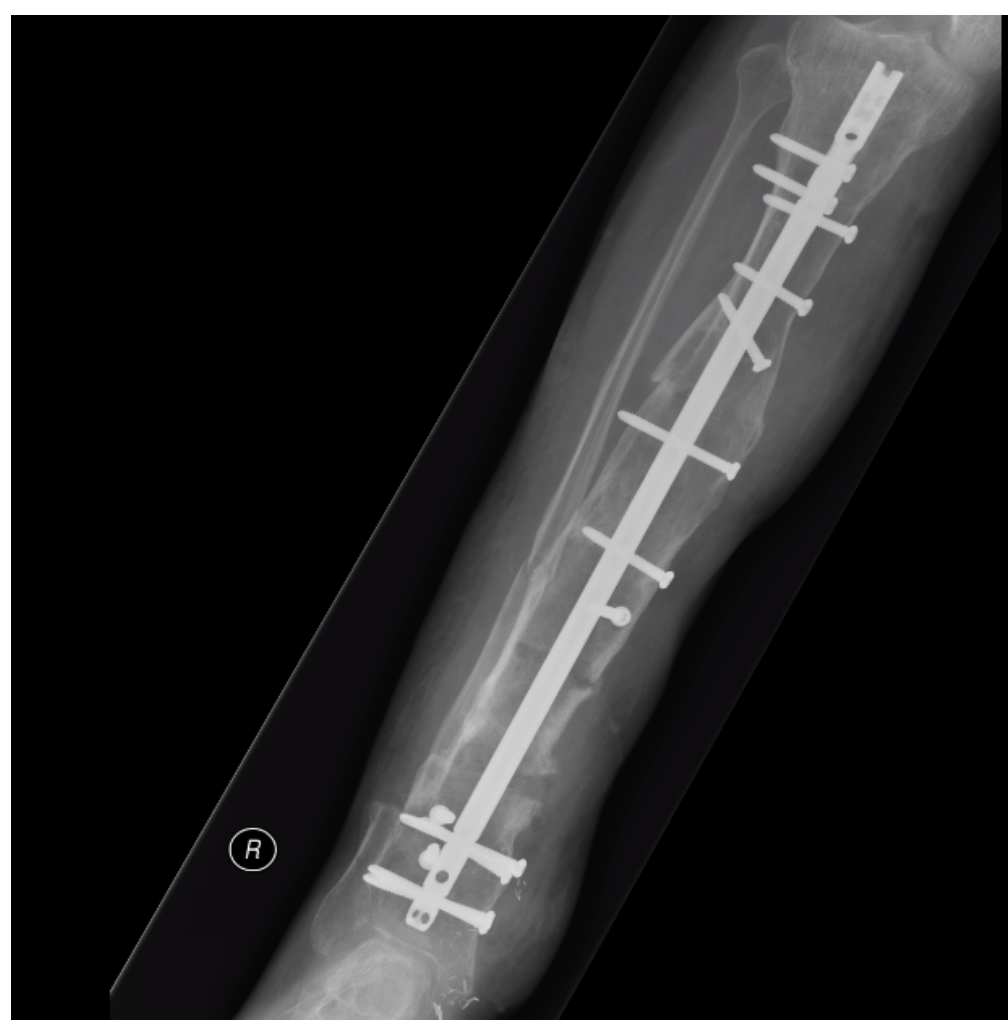

Figure 3: Final post op X-ray after deformity correction.

more and has returned to his full time occupation running multiple businesses.

\section{Conclusion}

There is very sparse literature describing the management of severe fibrous dysplasia in the tibia. A combined multidisciplinary approach to this rare disease and associated severe deformity has demonstrated excellent outcome for this patient.

\section{References}

1. Claudia E Dumitrescu, Michael T Collins (2008) McCuneAlbright syndrome. Orphanet Journal of Rare Diseases 3: 12.
2. Leet Al, Collins MT (2007) Current approach to fibrous dysplasia of bone and McCune-Albright syndrome. J Child Orthop 1: 3-17.

3. Hart ES, Kelly MH, Brillante B, Chen CC, Ziran N, et al. (2007) Onset, progression, and plateau of skeletal lesions in fibrous dysplasia and the relationship to functional outcome. J Bone Miner Res 22: 1468-1474.

4. Rivera Rosado E, Hernandez Ortiz E, Lopez Aponte C, Marrero Ortiz PV (2016) Bilateral tibia fibrous dysplasia in a pediatric patient treated with intramedullary nailing: $A$ case report. Bol Asoc Med P R 108: 23-25.

5. Stanton RP, Ippolito E, Springfield D, Lindaman L, Wientroub S, et al. (2012) The surgical management of fibrous dysplasia of bone. Orphanet J Rare Dis 7: S1. 